Board of Governors of the Federal Reserve System

International Finance Discussion Papers

Number 649

October 1999

HIGH FREQUENCY DATA, FREQUENCY DOMAIN INFERENCE AND VOLATILITY FORECASTING

Jonathan H. Wright and Tim Bollerslev

NOTE: International Finance Discussion Papers are preliminary materials circulated to stimulate discussion and critical comment. References in publications to International Finance Discussion Papers (other than an acknowledgment that the writer has had access to unpublished material) should be cleared with the author or authors. Recent IFDPs are available on the Web at www.bog.frb.fed.us. 


\title{
HIGH FREQUENCY DATA, FREQUENCY DOMAIN INFERENCE AND VOLATILITY FORECASTING
}

\author{
Jonathan H. Wright and Tim Bollerslev*
}

\begin{abstract}
While it is clear that the volatility of asset returns is serially correlated, there is no general agreement as to the most appropriate parametric model for characterizing this temporal dependence. In this paper, we propose a simple way of modeling financial market volatility using high frequency data. The method avoids using a tight parametric model, by instead simply fitting a long autoregression to log-squared, squared or absolute high frequency returns. This can either be estimated either by the usual time domain method, or alternatively the autoregressive coefficients can be backed out from the smoothed periodogram estimate of the spectrum of log-squared, squared or absolute returns. We show how this approach can be used to construct volatility forecasts, which compare favorably with some leading alternatives in an out-of-sample forecasting exercise.
\end{abstract}

Keywords: Autoregression; Spectrum; Volatility Forecasting; Wiener-Kolmogorov Filter; High Frequency Data; Exchange Rates.

${ }^{*}$ Bollerslev is a professor of economics at Duke University and Wright is an economist in the International Finance Division of the Federal Reserve Board. The first author would like to acknowledge the financial support by a grant from the NSF to the NBER. We are grateful to Jim Stock for helpful comments on an earlier draft, and to Olsen \& Associates for providing the high-frequency data. The usual disclaimer applies. 


\section{Introduction}

While asset returns generally appear to be close to a martingale difference sequence, there is overwhelming evidence that asset returns are not independently distributed over time, because of volatility clustering. Serial correlation in the volatility of asset returns has been documented in an enormous number of papers, going back to Mandelbrot (1963) and Fama (1965), and, more recently, the ARCH literature pioneered by Engle (1982). Recent survey articles include Bollerslev, Chou and Kroner (1992), Bollerslev, Engle and Nelson (1994) and Diebold and Lopez (1995). Many parametric models have been proposed for modeling this persistence of volatility in asset returns. These include the ARCH and GARCH models (Engle (1982) and Bollerslev (1986)), the EGARCH model (Nelson (1991)) and stochastic volatility models (Taylor (1986) and Andersen (1994)). Some researchers have proposed models with long memory in the volatility process (including Baillie, Bollerslev and Mikkelsen (1996), Breidt, Crato and de Lima (1998), Harvey (1998) and Robinson (1991)). Other authors, such as Engle and Lee (1993) have proposed models in which the volatility process has two components: one of which is nearly nonstationary, while the other is much less persistent. A number of papers have considered nonparametric approaches to representing time varying conditional heteroskedasticity (Pagan and Schwert (1990), Gallant, Rossi and Tauchen $(1992,1993))$. In these models, squared asset returns are modeled as a nonparametric function of lagged returns. However, in practice it 
is necessary to choose a relatively small number of lags, because of the well known problems in applying nonparametric methods to high dimensional models. When working with high frequency data, there are also important intradaily patterns in volatility (Andersen and Bollerslev (1997)). So, while it is clear that the volatility of asset returns is highly persistent, there is no consensus as to the best model for representing these volatility dynamics. This is especially true with high frequency data.

A different approach to modeling volatility dynamics is proposed in this paper, explicitly utilizing the additional information in high frequency data. The idea is to model volatility dynamics by fitting a long AR representation to log-squared, squared or absolute high frequency asset returns. This can be implemented by first estimating the spectrum of log-squared, squared or absolute returns and then using a numerical method, sometimes known as the Wiener-Kolmogorov filter, to solve for the coefficients in the corresponding AR representation. Alternatively, the AR representation may simply be estimated by the usual time domain method. These approaches are not nonparametric, but may allow for flexible dynamics. We emphasize that they are appropriate only in the presence of a very large sample of high frequency data, so that fitting very long autoregressions is both feasible and necessary to reasonably approximate the observed patterns of volatility clustering. Indeed, these methods fare poorly with a sample of daily returns, as shall be demonstrated below. In a re- 
lated context, Andersen and Bollerslev (1998) showed that intradaily data was vitally important in the meaningful ex-post evaluation of daily volatility forecasts. Moreover, Andersen, Bollerslev and Lange (1999) have recently shown that while the gains in forecast error accuracy from correctly specified high frequency GARCH volatility models can be very large from a theoretical perspective, the standard models tend to perform very poorly when applied directly to high frequency data. This paper shows how the high frequency data may easily be used to construct superior daily volatility forecasts.

The plan for the remainder of the paper is as follows. The proposed method for modeling volatility is introduced in the next section. In section 3, it is used to predict future values of the volatility of the Deutschemark-U.S. Dollar spot exchange rate based on a ten year sample of 5-minute returns. Section 4 concludes.

\section{Estimation of Volatility Dynamics}

\subsection{The Assumed Model}

We begin by making a high level primitive assumption that an appropriate proxy for the time series of volatilities, such as the log-squared returns, has an AR representation. Specifically, we assume that $\left\{y_{t}\right\}_{t=1}^{T}$ is a martingale difference sequence of asset returns such that

Assumption A1: $a(L)\left(\log \left(y_{t}^{2}\right)-\mu_{1}\right)=\varepsilon_{t}$. 
Here and throughout, $a(L)=1-a_{1} L-a_{2} L^{2} \ldots$ denotes a (possibly infinite order) lag polynomial such that $\sum_{j=0}^{\infty}\left|a_{j}\right| j^{1 / 2}<\infty^{1}, L$ is the lag operator, and $\varepsilon_{t}$ is an independently and identically distributed sequence with mean zero, variance $\sigma^{2}$, and finite fourth moment. As alternatives to assumption A1, we could instead specify that the squared or absolute returns have an AR representation, modified so that the squared or absolute returns cannot be negative ${ }^{2}$. To accommodate this we introduce alternative assumptions $\mathrm{Al}^{\prime}$ and $\mathrm{A} 1^{\prime \prime}$ :

Assumption A1': $a(L)\left(z_{t}-\mu_{2}\right)=\varepsilon_{t}$ and $y_{t}^{2}=\max \left(z_{t}, 0\right)$.

Assumption A1": $a(L)\left(z_{t}-\mu_{3}\right)=\varepsilon_{t}$ and $\left|y_{t}\right|=\max \left(z_{t}, 0\right)$.

Assumption A1 nests some standard models of stochastic volatility as special cases. For example, the standard autoregressive stochastic volatility (ARSV) model specifies that

$$
\begin{gathered}
y_{t}=\exp \left(h_{t} / 2\right) \sigma_{u} u_{t} \\
(1-\phi L) h_{t}=\sigma_{v} v_{t}
\end{gathered}
$$

where $u_{t}$ and $v_{t}$ are i.i.d. $\mathrm{N}(0,1)$ and $|\phi|<1$. This implies that $\log \left(y_{t}^{2}\right)=h_{t}+$ $\log \left(\sigma_{u}^{2}\right)+\log \left(u_{t}^{2}\right)$ which has a representation as an ARMA $(1,1)$ reduced form (see e.g.

\footnotetext{
$1 \frac{1}{2}$-summability of these coefficients is required to enable the spectrum of the log-squared data to be uniformly consistently estimable.

${ }^{2}$ It is of course an advantage of assumption A1 that no such modification is required.
} 
Harvey, Ruiz and Shephard (1994)) and assumption A1 is satisfied ${ }^{3}$.

One approach to estimating $a(L)$ is simply to fit an $A R(p)$ to the observed sample, where the order of the estimated autoregression, $p$, goes to infinity but at a rate slower than $T^{1 / 3}$, as the sample size $T$ goes to infinity ${ }^{4}$. Let the resulting estimates for $a_{1}, a_{2}, \ldots$ be denoted by $\tilde{a}_{1}, \tilde{a}_{2}, \ldots$. The Wiener-Kolmogorov filter provides an alternative approach to estimating these coefficients, which may potentially work better when $a(L)$ is not in fact a small order autoregressive polynomial.

\subsection{The Wiener-Kolmogorov Filter}

Suppose that assumption A1 holds and that the spectrum of $\log \left(y_{t}^{2}\right)-\mu_{1}$ is known. Call this spectrum $f(\lambda)$. Of course, the spectrum of any time series contains, in principle, the same information as its AR representation, and going from the AR representation to the spectrum is numerically straightforward, as $f(\lambda)=\frac{\sigma^{2}}{2 \pi}\left\|a\left(e^{i \lambda}\right)\right\|^{-2}$. Inverting this transformation is harder, but there is a standard result (see, for example, Brillinger (1981) p.79) which provides a closed form representation for the autoregressive coefficients in terms of the spectrum of a univariate time series. Specifically,

$$
a_{j}=\frac{1}{2 \pi} \int_{-\pi}^{\pi} B(\lambda)^{-1} \exp (i j \lambda) d \lambda
$$

where

\footnotetext{
${ }^{3}$ The fractionally integrated stochastic volatility model of Breidt, Crato and de Lima (1998) likewise yields an AR representation, but does not satisfy the requirement that $\sum_{j=0}^{\infty}\left|a_{j}\right| j^{1 / 2}<\infty$.

${ }^{4}$ see Berk (1974).
} 


$$
B(\lambda)=\exp \left(\frac{1}{2} c(0)+\sum_{v=1}^{\infty} c(v) \exp (-i v \lambda)\right)
$$

and

$$
c(v)=\frac{1}{2 \pi} \int_{-\pi}^{\pi} \log (f(\lambda)) \exp (i v \lambda) d \lambda
$$

This algorithm is sometimes referred to as the Wiener-Kolmogorov filter (Bhansali and Karavellas (1983)). Naturally, in empirical applications, no researcher ever knows the true spectrum of the log-squared returns. However, the spectrum may be estimated by smoothing the periodogram and the estimated spectrum may then be substituted into the Wiener-Kolmogorov filter. In particular, define the periodogram of the logsquared data as

$$
I(\lambda)=\frac{1}{2 \pi T}\left\|\Sigma_{t=1}^{T}\left(\log \left(y_{t}^{2}\right)-\hat{\mu}_{1}\right) e^{i t \lambda}\right\|^{2}
$$

where $\hat{\mu}_{1}$ denotes the sample average of $\log \left(y_{t}^{2}\right)$. The associated smoothed estimate of the spectrum is then given by

$$
\hat{f}(\lambda)=\frac{1}{T h} \Sigma_{k=-n}^{n} K\left(\frac{\lambda-\lambda_{k}}{h}\right) I\left(\lambda_{k}\right)
$$

where $n=[T / 2], \lambda_{k}=\frac{2 \pi k}{T}, \mathrm{~K}($.$) is a kernel function and h$ is a bandwidth parameter which converges to zero but at a rate slower than $O\left(T^{-1}\right)$. In this paper, we use the Epanechnikov kernel which sets

$$
K(\omega)=0.75\left(1-w^{2}\right) 1(|\omega| \leq 1)
$$


Under the given conditions, $\hat{f}(\lambda)$ is consistent for $f(\lambda)$ uniformly on $[-\pi, \pi]$ (Brillinger $\left.(1981)^{5}\right)$

Let the resulting estimates for $a_{1}, a_{2}, \ldots$ obtained by substituting this estimated spectrum into the Wiener-Kolmogorov filter in equations (1), (2) and (3) be denoted by $\hat{a}_{1}, \hat{a}_{2}, \ldots$. Because each of these coefficients is a continuous function of $f(\lambda)$, it follows, that if $\hat{f}(\lambda)$ is uniformly consistent for $f(\lambda)$, then each $\hat{a}_{j}$ is consistent for $a_{j}$. Under assumptions $\mathrm{A}^{\prime}$ or $\mathrm{A}^{\prime \prime}$, exactly the same approach may be used to solve for the AR coefficients of squared returns or absolute returns from the estimated spectrum of squared or absolute returns, respectively. The approach of estimating the spectrum by smoothing the periodogram and then backing out the implied AR coefficients has previously been used by Bhansali $(1973,1974,1977)$ among others. More recently a similar idea has also been applied by Wright (1999) in the context of impulse response analysis. The present paper shows how this frequency domain approach may be applied to modeling the volatility of asset returns ${ }^{6}$.

\footnotetext{
"Other kernels will, of course, also guarantee the uniform consistency of $\hat{f}(\lambda)$, as discussed by Brillinger (1981).

${ }^{6}$ As previously noted, long-memory in the volatility process is not strictly speaking allowed for, as we have assumed that the coefficients in $a(L)$ are $\frac{1}{2}$-summable. Concretely, if $f(\lambda)$ is not bounded away from zero at the origin, then $\hat{f}(\lambda)$ is not uniformly consistent, and even if we knew the true spectrum, the integral in (3) is not defined. Nevertheless, from a practical empirical perspective, the estimated $a(L)$ coefficients may get arbitrarily close to a true long-memory process.
} 


\subsection{The Modified Log-Squared Transformation.}

Assumption A1 applies to log-squared asset returns. An inlier problem often arises when dealing with the log-squared transformation; if the asset return is very close to zero, then the log-squared transformation yields a large negative number. Such an observation can then greatly affect the results of subsequent data analysis. In the extreme case, if the asset return is equal to zero, then the log-squared transformation is not even defined. Consequently, Fuller (1996) proposes a slight modification of the log-squared transformation, which does not converge to minus infinity as the argument converges to zero. This specifies that the transformed series of asset returns is

$$
y_{t}^{*}=\log \left(y_{t}^{2}+\tau s^{2}\right)-\frac{\tau s^{2}}{y_{t}^{2}+\tau s^{2}}
$$

where $s^{2}$ is the sample variance of $y_{t}$ and $\tau$ is a small constant. In all empirical work in this paper, we use $y_{t}^{*}$ with $\tau=0.02$, instead of the log-squared returns ${ }^{7}$. For convenience, we however adopt the shorthand of referring to $y_{t}^{*}$ as the log-squared returns.

\footnotetext{
${ }^{7}$ The choice of $\tau$ follows Fuller (1996) and Breidt and Carriquiry (1996).
} 


\section{Forecasting Integrated Volatility}

\subsection{Integrated Volatility and Alternative Volatility Measures}

A leading motivation for studying models of time-varying conditional heteroskedasticity is to be able to forecast future volatility. One common measure of the quality of a forecast of an arbitrary variable, $x_{t}$, is the R-squared in a regression of the ex-post realized values of $x_{t}$ on its forecast values (and a constant). We refer to this procedure as the Mincer-Zarnowitz method (Mincer and Zarnowitz (1969)). The R-squared in the Mincer-Zarnowitz regression indicates that GARCH and other standard volatility models provide poor forecasts of future squared returns (see, e.g. Jorion (1995), Andersen and Bollerslev (1998)).

However, the squared one-period return is generally a very noisy measure of the true latent volatility and is, as such, virtually unforecastable. Meanwhile if the researcher is interested in the volatility of the return of an asset over any fixed time period from $t_{0}$ to $t_{1}$ (such as a day, or the life of an option) and if the researcher has access to high frequency intradaily data on the asset returns, then the squared high frequency returns, summed over the period from $t_{0}$ to $t_{1}$, constitute a much more accurate estimate of the true ex-post volatility of the returns over that fixed time period ${ }^{8}$. We refer to this measure as the integrated volatility. In a related

\footnotetext{
${ }^{8}$ As discussed more formally in Andersen, Bollerslev, Diebold and Labys (1999), if the returns follow a special semimartingale, the quadratic variation of the process constitutes a natural measure of the ex-post realized volatility.
} 
context, Andersen and Bollerslev (1998) point out that standard GARCH models provide good forecasts of future values of integrated volatility. In particular they show that the R-squared in the Mincer-Zarnowitz regression is quite high, for some standard datasets of asset returns. In addition to allowing for more meaningful expost volatility forecast evaluation, this integrated volatility measure also corresponds directly to the theoretical notion of volatility entertained in the diffusion models proposed by Barndorff-Nielsen and Shephard (1998). This same measure also figures prominently in the stochastic volatility option pricing literature (e.g. Hull and White (1987)) and its formal estimation has recently been explored by Gallant, Hsu and Tauchen (1998).

In this section, we consequently focus on forecasting integrated volatility. For concreteness, and to tie in with the dataset analyzed below, let $y_{t}$ denote a 5-minute return series. With 24 hour markets, there are 2885 -minute observations in a day. It is also convenient to use the notation $y_{s, n}$ to refer to the nth 5 -minute return on day $s, s=1, \ldots S, n=1, \ldots 288$. Clearly, $y_{t}=y_{s, n}$ with $t=288(s-1)+n$. The integrated volatility over the day $s$ is then defined as $V_{I}(s)=\frac{1}{288} \sum_{n=1}^{288} y_{s, n}^{2}$.

\subsection{The data}

The spot Deutschemark-U.S. Dollar exchange rate data were collected and provided by Olsen and Associates in Zürich, Switzerland. The full sample spans the period from December 1, 1986 through December 1, 1996. The returns are calculated as the 
difference between the linearly interpolated average of the mid-point of the logarithmic bid and ask for the two nearest quotes, resulting in a total of 2885 -minute return observations per day ${ }^{9}$. Although the foreign exchange market is officially open 24 hours a day and 365 days a year, the trading activity slows decidedly during the weekend period. In order to avoid confounding the evidence by such weekend patterns, we simply excluded all returns from Friday 21:00 Greenwich Mean Time (GMT) through Sunday 21:00 GMT; a similar weekend no-trade convention was adopted by Andersen and Bollerslev (1997). Furthermore, the market slows decidedly over certain holiday periods. Excluding the most important of these days ${ }^{10}$, leaves us with a sample of 2,445 complete days, for a total of $T=2,445 \times 288=704,1605$-minute $y_{t}$ return observations.

Consistent with the notion of efficient markets, the 5-minute returns are approximately mean zero and serially uncorrelated. At the same time, the evidence for volatility clustering is overwhelming. For instance, the lag-1 sample autocorrelation coefficient for the squared 5-minute returns equals 0.195 , which is overwhelmingly significant at any level. The autocorrelograms of the squared, log-squared and absolute returns, in Figure 1, all show a rapid initial decay but then decay only slowly.

\footnotetext{
${ }^{9}$ See Dacorogna et al. (1993) and Müller et al. (1990) for a more detailed description of the activity patterns in the foreign exchange market and the method of data capturing and filtering that underlie the return calculations.

${ }^{10}$ For further discussion of the specific exclusions, we refer to Andersen, Bollerslev, Diebold and Labys (1999), where the same data is analyzed from a different perspective.
} 
Additionally, these autocorrelograms have a distinct seasonal pattern. Similar periodic autocorrelograms for other speculative returns and time periods have previously been reported in the literature by Dacorogna et al. (1993) and Andersen and Bollerslev (1997) among others, who attribute the periodicities to the existence of strong intradaily volatility patterns associated with the opening and closing of the various financial centers around the world.

\subsection{Volatility forecasts with daily data}

Arguably, the most common approach to volatility forecasting is based on the estimation of daily GARCH models. Specifically, let $y_{s}^{(D)}=\sum_{n=1}^{288} y_{s, n}$ denote the daily return for day $s$. The $\operatorname{GARCH}(p, q)$ model then specifies that

$$
\begin{gathered}
y_{s}^{(D)}=\sigma_{s} \eta_{s} \\
\sigma_{s}^{2}=\omega+\sum_{i=1}^{p} \alpha_{i} \sigma_{s-i}^{2} \eta_{s-i}^{2}+\sum_{j=1}^{q} \beta_{j} \sigma_{s-j}^{2}
\end{gathered}
$$

where $\eta_{s}$ is serially uncorrelated with mean zero and variance one, $\omega>0, \alpha_{i} \geq 0$ and $\beta_{j} \geq 0$, and the parameters satisfy the conditions in Nelson and Cao (1992) for $\sigma_{s}^{2}$ to be positive (almost surely). The quasi-maximum likelihood estimates of these parameters, obtained under the auxiliary assumption of conditional normality, may be calculated and the associated one day ahead forecast of volatility on day $s$ can be viewed as a forecast of $V_{I}(s)$. This forecast can then be evaluated in terms of its mean square prediction error, or in terms of the R-squared, coefficient estimates and 
their standard errors in the Mincer-Zarnowitz regression.

Table 1 shows the resulting quasi-maximum likelihood estimates of the parameters of $\operatorname{GARCH}(1,1), \operatorname{GARCH}(1,2), \operatorname{GARCH}(2,1)$ and $\operatorname{GARCH}(2,2)$ models, estimated using the full sample of 2,445 days ${ }^{11}$. Table 2 shows the out-of-sample forecast evaluation criteria for these specifications, estimating the model parameters with the first half of the data and evaluating the forecasts over the remainder of the sample. In Table 2, we also report the forecast evaluation criteria for an $\operatorname{EGARCH}(1,1)$ model and for forecasts constructed by simply fitting an $\mathrm{AR}(10)$ to daily squared returns. In Table 2, we can see that the $\operatorname{GARCH}(1,1)$ and $\operatorname{EGARCH}(1,1)$ models ${ }^{12}$ provide the best forecasts. The $\operatorname{GARCH}(1,1)$ forecasts of integrated volatility have an R-squared in the Mincer-Zarnowitz regression similar to that found by Andersen and Bollerslev (1998). Meanwhile, simply fitting an AR(10) to the daily squared returns produces very poor out-of-sample forecasts ${ }^{13}$. Thus, it is clear that simple parametric GARCH models produce better forecasts than just fitting an autoregression to the squared returns, when working with daily data.

\footnotetext{
${ }^{11}$ Among all GARCH(p,q) models for daily data with $p, q \leq 3$, the Akaike and Schwartz information criteria both selected the $\operatorname{GARCH}(2,1)$ specification.

${ }^{12}$ Results for some higher EGARCH models are not shown, but these generate less good forecasts, especially out-of-sample.

${ }^{13}$ This result is not sensitive to the order of the autoregression that is fitted.
} 


\subsection{Volatility Forecasts using the Intradaily Data}

We now turn to volatility forecasts, explicitly constructed from the 5-minute returns.

One forecasting strategy is to start with assumption $\mathrm{Al}^{\prime}$, estimate the spectrum of $v_{t}=y_{t}^{2}-\hat{\mu}_{2}$ (where $\hat{\mu}_{2}$ denotes the sample mean of $\left.y_{t}^{2}\right)$ and then use the WienerKolmogorov filter to calculate $\left\{\hat{a}_{j}\right\}$, the associated estimates of $\left\{a_{j}\right\}$. Let $\hat{v}_{t+k \mid t}$ denote the resulting forecast of $v_{t+k}$ given $v_{t}$ and lagged values, as given by the recursions

$$
\hat{v}_{t+1 \mid t}=\sum_{j=1}^{\infty} \hat{a}_{j} v_{t-j}
$$

and

$$
\hat{v}_{t+k \mid t}=\sum_{j=1}^{k-1} \hat{a}_{j} \hat{v}_{t+k-j \mid t}+\sum_{j=k}^{\infty} \hat{a}_{j} v_{t+k-j}
$$

The integrated volatility at date $s, V_{I}(s)$, conditional on returns from day $s-1$ and earlier may then be forecast ${ }^{14}$ as

$$
\hat{V}_{I}(s)=\frac{1}{288} \sum_{n=1}^{288} \max \left(\hat{\mu}_{2}+\hat{v}_{288(s-1)+n \mid 288(s-1)}, 0\right)
$$

Forecasts of $V_{I}(s)$ may equally be formed using $\left\{\tilde{a}_{j}\right\}$, the estimates of the AR coefficients obtained from the usual time domain autoregression. Let this forecast be denoted $\tilde{V}_{I}(s)$

Table 3 shows the forecast evaluation criteria for $\hat{V}_{I}(s)$ and $\tilde{V}_{I}(s)$ in the outof-sample forecasting exercise ${ }^{15}$. In constructing these forecasts, the spectrum of

\footnotetext{
${ }^{14}$ In the empirical work, we truncated the infinite sum in (7) after 10,000 terms; though the results are virtually identical using just 5,000 terms.

${ }^{15}$ Counterparts of Tables 2 and 3 for in-sample forecasts and for the Japanese Yen - U.S. Dollar exchange rate are similar, and are available on request.
} 
the squared returns was estimated using a bandwidth $h=0.0009$, while the fitted time domain autoregression was of order 2,050. These parameters were chosen so as to minimize the out-of-sample mean square prediction error. This leaves open the question of how a researcher should select these parameters in practice, but ensures that we can make a fair comparison between $\hat{V}_{I}(s)$ and $\tilde{V}_{I}(s)$.

Assumption $\mathrm{Al}^{\prime}$ is the natural starting point for forecasting integrated volatility as it relates directly to the squared returns. Alternatively, assumption A1 or A1" can be used to justify the corresponding forecasts of the future log-squared or absolute 5minute returns. These forecasts may then in turn be transformed into ad hoc forecasts of the future squared 5-minute returns by exponentiating or squaring the forecasts, followed by multiplication by a scaling factor ${ }^{16}$. In the case of assumption A1, the scaling factor is the ratio of the mean of the squared returns to the exponent of the mean of the log-squared returns. In the case of assumption $\mathrm{A1}^{\prime \prime}$, it is the ratio of the mean of the squared returns to the square of the mean of the absolute returns. The forecasts of future squared 5-minute returns can be summed up to obtain forecasts of future integrated volatility, based on assumptions A1 or A1". These forecasts are ad $h o c$, but their practical usefulness is an empirical question. Of course, these forecasts could alternatively be based on fitting a long autoregression to the log-squared or the

\footnotetext{
${ }^{16}$ The scaling factor is a simple attempt to correct for the fact that the expectation of a nonlinear function of a random variable is not equal to the nonlinear function of the expectation of that random variable.
} 
absolute returns in the time domain. Table 3 also shows the results for these forecasts, which we refer to (in obvious notation) as $\hat{V}_{I}^{L O G-S Q}(s), \tilde{V}_{I}^{L O G-S Q}(s), \hat{V}_{I}^{A B S}(s)$ and $\tilde{V}_{I}^{A B S}(s)$.

Table 3 also shows the results for the forecasts of daily integrated volatility obtained by fitting an $\mathrm{AR}(10)$ model directly to the daily integrated volatility, $V_{I}(s)$. This is still a forecast based on high frequency data in the sense that it cannot be constructed by a researcher who only has access to daily data. As a final comparison, we also used GARCH models fitted directly to the high frequency 5-minute returns to construct the daily volatility forecasts. However, to conserve space, we omit these results, as they yielded very unreasonable forecasts. Consistent with the earlier findings in Andersen, Bollerslev and Lange (1999), a small order GARCH model is grossly underparameterized as a model of intradaily asset returns ${ }^{17}$. For each forecast in Tables 2 and 3, we also tested the significance of the difference in the mean square prediction error between that forecast and the forecast obtained from the commonly employed daily GARCH$(1,1)$ model using the procedure described in Diebold and Mariano (1995).

The forecast evaluation criteria for $\hat{V}_{I}(s)$ and $\tilde{V}_{I}(s)$ are very similar, though the frequency domain forecasts give a slightly higher R-squared in the Mincer-Zarnowitz

\footnotetext{
${ }^{17}$ For instance, fitting a $\operatorname{GARCH}(1,1)$ model to 5 -minute returns, the sum of the GARCH coefficients was 1.04 and the in-sample mean square prediction error of the associated forecasts was $2.4 \times 10^{6}$ !
} 
regressions. Meanwhile, both work much better than any of the forecasts in Table 2, based on daily data alone. The out-of-sample mean square prediction error of $\hat{V}_{I}(s)$ is $19 \%$ below that of the standard $\operatorname{GARCH}(1,1)$ forecast; this improvement in forecasting performance is highly significant. Among the forecasts based on fitting autoregressions to $\log$-squared and absolute returns, the frequency domain forecasts consistently have a slight edge over the time domain forecasts. Also $\hat{V}_{I}^{L O G-S Q}(s)$, $\tilde{V}_{I}^{L O G-S Q}(s)$ do less well than $\hat{V}_{I}(s)$ and $\tilde{V}_{I}(s)$, while $\hat{V}_{I}^{A B S}(s), \tilde{V}_{I}^{A B S}(s)$ do better than $\hat{V}_{I}(s)$ and $\tilde{V}_{I}(s)$ (notwithstanding their more ad hoc justification ${ }^{18}$ ). The out-ofsample mean square prediction error of $\hat{V}_{I}^{A B S}(s)$, the best of all the forecasts, is $20 \%$ below that of the standard daily $\operatorname{GARCH}(1,1)$ forecast. Simply fitting an autoregression to $V_{I}(s)$ produces forecasts which are clearly superior to any of the forecasts in Table 2, though yields a lower R-squared in the Mincer-Zarnowitz regression than any of the other forecasts using intradaily data in Table 3 .

\footnotetext{
${ }^{18}$ We attribute the good performance of the forecasts based on 5-minute absolute returns to the fact that absolute returns are relatively outlier-resistant.
} 


\section{Concluding Remarks}

In this paper, we have proposed modeling volatility dynamics with high frequency data by simply fitting an autoregression to log-squared, squared or absolute returns. This autoregression can be estimated in the usual way, or can be backed out from a nonparametric smoothed periodogram estimate of the spectrum. We conclude that, when working with high frequency intradaily data, these simple autoregressions tend to work better in forecasting future volatility than standard GARCH and EGARCH models, fitted either to daily or intradaily data. Overall, this general conclusion is not very sensitive to whether the autoregressions are estimated in the time domain or in the frequency domain, although the latter procedure results in the lowest mean square prediction error. In sum, Andersen and Bollerslev (1998) showed that intradaily data was vitally important in the meaningful ex-post evaluation of daily volatility forecasts; this paper shows how the high frequency data may easily be used to construct superior daily volatility forecasts. Meanwhile, the approach to modeling volatility dynamics advocated here can of course be used in applications other than volatility forecasting; for example, it could be used to construct bootstrap distributions for test statistics. It will be interesting to further explore these issues in future research. 


\section{References}

Andersen, T.G. (1994): Stochastic Autoregressive Volatility: A Framework for Volatility Modeling, Mathematical Finance, 4, pp.75-102.

Andersen, T.G. and T. Bollerslev (1997): Intraday Periodicity and Volatility Persistence in Financial Markets, Journal of Empirical Finance, 4, pp.115-158.

Andersen, T.G. and T. Bollerslev (1998): Answering the Skeptics: Yes, Standard Volatility Models do Provide Accurate Forecasts, International Economic Review, 39, pp.885-905.

Andersen, T.G., T. Bollerslev, F.X. Diebold and P. Labys (1999): The Distribution of Exchange Rate Volatility, unpublished manuscript.

Andersen, T.G., T. Bollerslev and S. Lange (1999): Forecasting Financial Market Volatility: Sample Frequency vis-a-vis Forecast Horizon, Journal of Empirical Finance, forthcoming.

Baillie, R.T.,T. Bollerslev and H.O. Mikkelsen (1996): Fractionally Integrated Generalized Autoregressive Conditional Heteroskedasticity, Journal of Econometrics, 74, pp.3-30.

Barndorff-Nielsen, O.E. and N. Shephard (1998): Continuous Time Volatility: Model Construction and Inference, unpublished manuscript.

Berk, K.N. (1974): Consistent Autoregressive Spectral Estimates, Annals of Statistics, 2, pp.489-502.

Bhansali, R.J. (1973): A Monte Carlo Comparison of the Regression Method and the Spectral Methods of Prediction, Journal of the American Statistical Association, 68, pp.621-625.

Bhansali, R.J. (1974): Asymptotic Properties of the Wiener-Kolmogorov Predictor I, Journal of the Royal Statistical Society, Series B, 36, pp.61-73.

Bhansali, R.J. (1977): Asymptotic Properties of the Wiener-Kolmogorov Predictor II, Journal of the Royal Statistical Society, Series B, 39, pp.66-72.

Bhansali, R.J. and D. Karavellas (1983): "Wiener Filtering (With Emphasis on Frequency-Domain Approaches)" in Handbook of Statistics, Vol. 3, (eds. D.R. Brillinger and P.R. Krishnaiah), Elsevier, Amsterdam.

Bollerslev, T. (1986): Generalized Autoregressive Conditional Heteroskedasticity, Journal of Econometrics, 31, pp.307-327.

Bollerslev, T., R.Y. Chou and K.F. Kroner (1992): ARCH Modeling in Finance: A Review of the Theory and Empirical Evidence, Journal of Econometrics, 52, pp.5-59. Bollerslev, T., R.F. Engle and D.B. Nelson (1994): "ARCH Models", in Handbook of Econometrics, Vol. 4, (eds. R.F. Engle and D.L. McFadden), Elsevier, Amsterdam.

Breidt, F.J., N. Crato and P. de Lima (1998): The Detection and Estimation of LongMemory in Stochastic Volatility, Journal of Econometrics, 83, pp.325-348. 
Breidt, F.J. and A.L. Carriquiry (1996): "Improved Quasi-Maximum Likelihood Estimators for Stochastic Volatility Models" in Modeling and Prediction: Honouring Seymour Geisel, (eds. A. Zellner and J.S. Lee), Springer, New York.

Brillinger, D.R. (1981): Time Series: Data Analysis and Theory, Holden-Day, San Francisco.

Dacorogna, M.M., U.A. Müller, R.J. Nagler, R.B. Olsen and O.V. Pictet (1993): A Geographical Model for the Daily and Weekly Seasonal Volatility in the Foreign Exchange Market, Journal of International Money and Finance, 12, pp.413-438.

Diebold, F.X. and J.A. Lopez (1995): "Modeling Volatility Dynamics" in Macroeconometrics: Developments, Tensions and Prospects (ed. K. Hoover), Kluwer Academic Press, Boston.

Diebold, F.X. and R.S. Mariano (1995): Comparing Predictive Accuracy, Journal of Business and Economic Statistics, 13, pp.253-263.

Engle, R.F. (1982): Autoregressive Conditional Heteroskedasticity with Estimates of the Variance of U.K. Inflation, Econometrica, 50, pp.987-1008.

Engle, R.F. and G. Lee (1993): A Permanent and Transitory Component Model of Stock Return Volatility, unpublished manuscript.

Fama, E. (1965): The Behavior of Stock Prices, Journal of Business, 38, pp.34-105.

Fuller, W. A. (1996): Introduction to Statistical Time Series, Wiley, New York.

Gallant, A.R., P.E. Rossi and G.E. Tauchen (1992): Stock Prices and Volume, Review of Financial Studies, 5, pp.199-242.

Gallant, A.R., P.E. Rossi and G.E. Tauchen (1993): Nonlinear Dynamic Structures, Econometrica, 61, pp.871-907.

Gallant, A.R., C.T. Hsu and G.E. Tauchen (1998): Calibrating Volatility Diffusions and Extracting Integrated Volatility, unpublished manuscript.

Harvey, A.C., E. Ruiz and N.G. Shephard (1994): Multivariate Stochastic Variance Models, Review of Economic Studies, 61, pp.247-264.

Harvey, A.C. (1998): "Long-Memory in Stochastic Volatility", in Forecasting Volatility in Financial Markets, (eds. J. Knight and S. Satchell), Butterworth-Heineman, London.

Hull, J. and A. White (1987): The Pricing of Options on Assets with Stochastic Volatility, Journal of Finance, 42, pp.381-400.

Jorion, P. (1995): Predicting Volatility in the Foreign Exchange Market, Journal of Finance, 50, pp.507-528.

Mandelbrot, B.B. (1963): The Variation of Certain Speculative Prices, Journal of Business, 36, pp.394-419.

Mincer, J. and V. Zarnowitz (1969): "The Evaluation of Economic Forecasts" in Economic Forecasts and Expectations (ed. J. Mincer), National Bureau of Economic Research, Cambridge. 
Müller, U.A., M.M. Dacorogna, R.B. Olsen, O.V. Pictet, M. Schwarz and C. Morgenegg (1990): Statistical Study of Foreign Exchange Rates, Empirical Evidence of a Price Change Scaling Law, and Intraday Analysis, Journal of Banking and Finance, 14, pp.1189-1208.

Nelson, D.B. (1991): Conditional Heteroskedasticity in Asset Returns: A New Approach, Econometrica, 59, pp.347-370.

Nelson, D.B. and C.Q. Cao (1992): Inequality Constraints in the Univariate GARCH Model, Journal of Business and Economic Statistics, 10, pp.229-235.

Pagan, A.R. and G.W. Schwert (1990): Alternative Models for Conditional Stock Volatility, Journal of Econometrics, 45, pp. 267-290.

Robinson, P.M. (1991): Testing for Strong Serial Correlation and Dynamic Conditional Heteroskedasticity in Multiple Regression, Journal of Econometrics, 47, pp.6784.

Taylor, S.J. (1986): Modeling Financial Time Series, Wiley, Chichester.

Wright, J.H. (1999): Frequency Domain Inference for Univariate Impulse Responses, Economics Letters, 63, pp.269-277. 
Table 1

Quasi Maximum Likelihood GARCH Parameter Estimates with Daily Data (standard errors in parenthesis)

\begin{tabular}{lllll}
\hline Parameter & GARCH$(1,1)$ & GARCH $(1,2)$ & GARCH $(2,1)$ & GARCH $(2,2)$ \\
\hline$\omega$ & 0.0069 & 0.0049 & 0.0072 & 0.0125 \\
& $(0.0017)$ & $(0.0021)$ & $(0.0017)$ & $(0.0034)$ \\
$\alpha_{1}$ & 0.0449 & 0.0313 & 0.0313 & 0.0472 \\
& $(0.0054)$ & $(0.0119)$ & $(0.0158)$ & $(0.0053)$ \\
$\alpha_{2}$ & & & 0.0151 & 0.0424 \\
& & & $(0.0165)$ & $(0.0054)$ \\
$\beta_{1}$ & 0.9414 & 1.2819 & 0.9395 & -0.0564 \\
& $(0.0075)$ & $(0.2709)$ & $(0.0077)$ & $(0.0075)$ \\
$\beta_{2}$ & & -0.3229 & & 0.9423 \\
& & $(0.2560)$ & & $(0.0072)$ \\
AIC & 2.0579 & 2.0584 & 2.0585 & 2.0561 \\
SIC & 2.0650 & 2.0679 & 2.0680 & 2.0679 \\
& & & & \\
\hline
\end{tabular}

Notes: This table reports the quasi maximum likelihood estimates of the GARCH parameters using the full 2,445 days of daily data, along with the associated standard errors. The Akaike and Schwartz information criteria (AIC and SIC) are also included. 
Table 2

Out-of-sample Properties of Alternative Estimates of $V_{I}(s)$ using daily data

\begin{tabular}{|c|c|c|c|c|}
\hline Forecasting Method & $\hat{b}_{0}$ & $\hat{b}_{1}$ & MSE & $R^{2}$ \\
\hline $\operatorname{GARCH}(1,1)$ & $\begin{array}{c}-0.322 \\
(0.033)\end{array}$ & $\begin{array}{l}1.758 \\
(0.062)\end{array}$ & 0.180 & 39.5 \\
\hline $\operatorname{GARCH}(1,2)$ & $\begin{array}{l}-0.145 \\
(0.034)\end{array}$ & $\begin{array}{l}1.379 \\
(0.064)\end{array}$ & $0.197^{\dagger}$ & 27.4 \\
\hline $\operatorname{GARCH}(2,1)$ & $\begin{array}{l}-0.249 \\
(0.034)\end{array}$ & $\begin{array}{l}1.606 \\
(0.065)\end{array}$ & $0.189^{\dagger}$ & 33.3 \\
\hline $\operatorname{GARCH}(2,2)$ & $\begin{array}{l}-0.013 \\
(0.028)\end{array}$ & $\begin{array}{l}1.125 \\
(0.050)\end{array}$ & 0.188 & 29.1 \\
\hline $\operatorname{EGARCH}(1,1)$ & $\begin{array}{c}-0.219 \\
(0.033)\end{array}$ & $\begin{array}{l}1.571 \\
(0.064)\end{array}$ & $0.190 \dagger$ & 32.7 \\
\hline $\operatorname{AR}(10)$ fitted to $y_{t}^{(D) 2}$ & $\begin{array}{l}-0.305 \\
(0.049)\end{array}$ & $\begin{array}{l}1.690 \\
(0.095)\end{array}$ & $0.218 \dagger$ & 20.7 \\
\hline
\end{tabular}

Notes: The table reports the estimated intercept and slope coefficients from the MincerZarnowitz regression, $\hat{b}_{0}$ and $\hat{b}_{1}$, respectively, along with their estimated standard errors (in parentheses), for all of the alternative forecasting procedures. The table also reports the mean square prediction error for each of these forecasts and the percentage R-squared from the Mincer-Zarnowitz regression. The superscript ${ }^{*}$ indicates that the mean square prediction error is significantly lower than that of the $\operatorname{GARCH}(1,1)$ forecast, while the superscript $\dagger$ indicates that it is significantly higher (at the $5 \%$ level, using the test of Diebold and Mariano (1995)). The details of the construction of these alternative volatility forecasts are provided in the text. The GARCH and EGARCH models are all estimated using the daily data $y_{t}^{(D)}$. All parameters are estimated using the first 1,222 days of data, while the forecasts are evaluated using the remaining 1,223 days in the sample. 
Table 3

Out-of-sample Properties of Alternative Estimates of $V_{I}(s)$ using 5-minute data

\begin{tabular}{|c|c|c|c|c|}
\hline Forecasting Method & $\hat{b}_{0}$ & $\hat{b}_{1}$ & MSE & $R^{2}$ \\
\hline $\begin{array}{l}\tilde{V}_{I}(s) \\
\text { (time domain) }\end{array}$ & $\begin{array}{l}-0.131 \\
(0.023)\end{array}$ & $\begin{array}{l}1.257 \\
(0.039)\end{array}$ & $0.145^{*}$ & 46.5 \\
\hline $\begin{array}{l}\tilde{V}_{I}^{L O G-S Q}(s) \\
\text { (time domain) }\end{array}$ & $\begin{array}{l}-0.158 \\
(0.024)\end{array}$ & $\begin{array}{l}0.954 \\
(0.030)\end{array}$ & 0.179 & 45.7 \\
\hline $\begin{array}{l}\tilde{V}_{I}^{A B S}(s) \\
\text { (time domain) }\end{array}$ & $\begin{array}{l}-0.042 \\
(0.020)\end{array}$ & $\begin{array}{l}0.900 \\
(0.027)\end{array}$ & $0.148^{*}$ & 48.1 \\
\hline $\begin{array}{l}\hat{V}_{I}(s) \\
\text { (frequency domain) }\end{array}$ & $\begin{array}{l}-0.182 \\
(0.024)\end{array}$ & $\begin{array}{l}1.356 \\
(0.041)\end{array}$ & $0.146^{*}$ & 47.2 \\
\hline $\begin{array}{l}\hat{V}_{I}^{L O G-S Q}(s) \\
\text { (frequency domain) }\end{array}$ & $\begin{array}{l}-0.216 \\
(0.026)\end{array}$ & $\begin{array}{l}1.059 \\
(0.033)\end{array}$ & 0.171 & 46.3 \\
\hline $\begin{array}{l}\hat{V}_{I}^{A B S}(s) \\
\text { (frequency domain) }\end{array}$ & $\begin{array}{l}-0.066 \\
(0.021)\end{array}$ & $\begin{array}{l}0.955 \\
(0.028)\end{array}$ & $0.144^{*}$ & 48.5 \\
\hline $\operatorname{AR}(10)$ fitted to $V_{I}(s)$ & $\begin{array}{l}-0.122 \\
(0.023)\end{array}$ & $\begin{array}{l}1.241 \\
(0.039)\end{array}$ & $0.156^{*}$ & 45.9 \\
\hline
\end{tabular}

Notes: As for Table 2. 
Figure 1: Autocorrelogram of Volatility Measures for DM-Dollar Returns
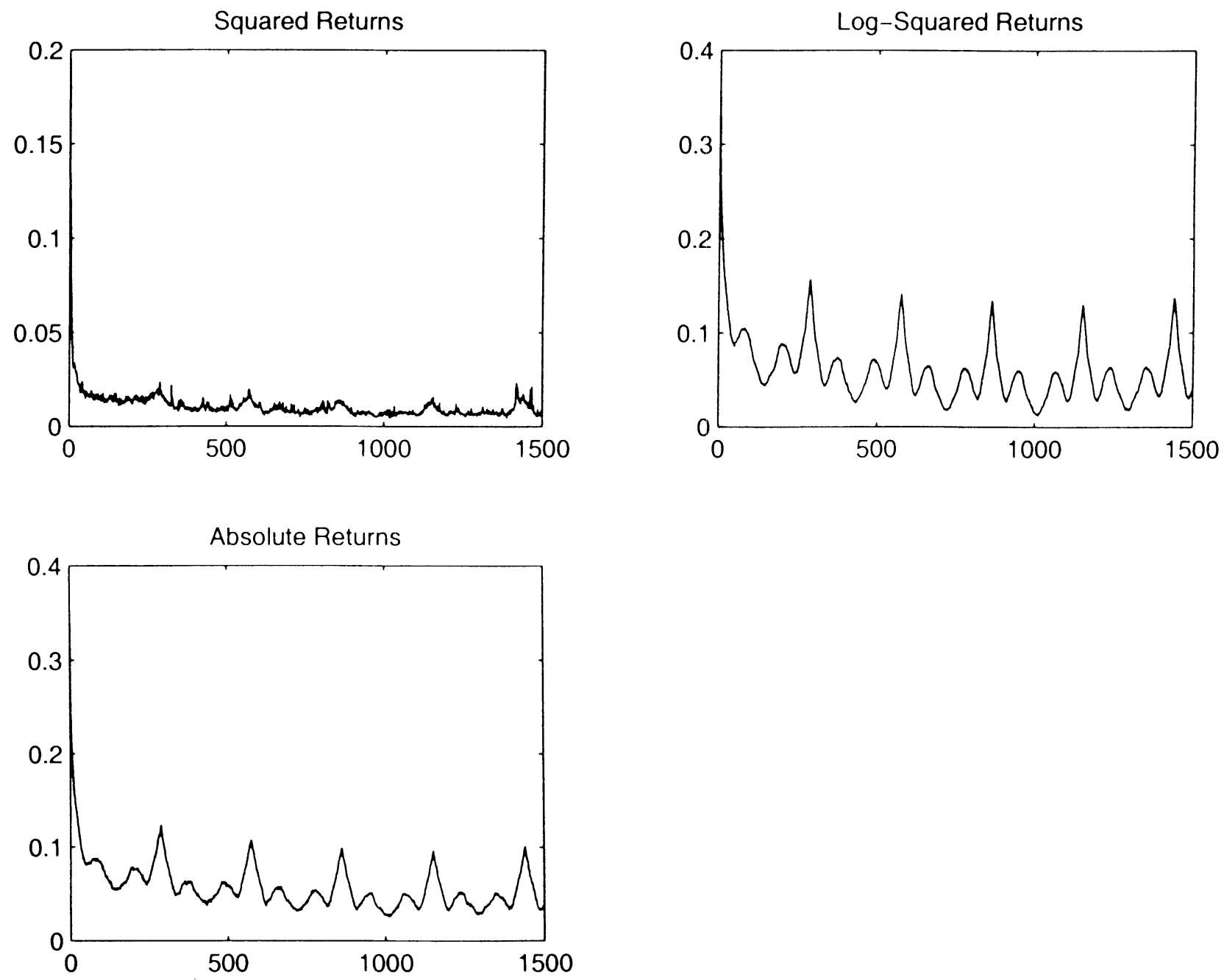

Notes: This figure gives the autocorrelogram of the squared, log-squared and absolute 5-minute demeaned DM-\$ returns, as described in the text. 